\title{
Electrical Control of Magnetic States
}

\author{
J. BARNAŚ \\ Department of Physics, Adam Mickiewicz University, Umultowska 85, 61-614 Poznań, Poland \\ and \\ Institute of Molecular Physics, Polish Academy of Sciences, M. Smoluchowskiego 17, 60-179 Poznań, Poland

\begin{abstract}
Charge and spin transport in artificial magnetic structures, like nanopillar spin valves, planar tunnel junctions, and mesoscopic double-barrier junctions (single-electron transistors) are briefly surveyed, with particular emphasis on magnetoresistance effects and current-induced magnetic switching and spin dynamics. The methods of spin current generation are also discussed, particularly the method based on spin Hall effect.
\end{abstract}

PACS numbers: 75.60.Ch, 75.70.Cn, 72.25.-b

\section{Introduction}

An important step in the search for novel artificial magnetic materials was the discovery of interlayer exchange coupling in $3 d$-metallic magnetic multilayers [1], and understanding of its basic features, like variation of the coupling parameter with the spacer [2] and magnetic layer [3] thicknesses. A breakthrough came up later (in 1988) with the discovery of giant magnetoresistance (GMR) $[4,5]$. Transport of spin and charge in magnetic multilayers and in other magnetic nanostructures became then of great interest from both fundamental and application points of view.

The GMR effect is a consequence of the existence of two well-defined and non-equivalent spin channels for electronic transport in ferromagnets [6]. This nonequivalence of spin channels has further consequences for transport properties of spatially nonuniform magnetic structures. Apart from the magnetoresistance phenomena (like the GMR effect), charge current in such systems is associated with spin current, and the latter can lead to magnetization switching between two stable magnetic states $[7-10]$ or to stationary precessional states $[11,12]$.

Similar phenomena also appear in other artificial magnetic systems, for instance in planar single-barrier tunnel junctions, where tunnel magnetoresistance [13] as well as current induced magnetic switching [14-17] have been experimentally observed. These effects also occur in both planar [18] and mesoscopic double-barrier tunnel junctions. In the latter case the central part of a double junction can be in the form of a metallic nanoparticle [19-21], semiconductor quantum dot [22, 23], or a molecule [24]. Of particular interest are double-barrier junctions based on magnetic molecules, where a pulse of spin-dependent current flowing through the molecule can switch the molecule's spin between two states of lowest energy [25].

The spin current is thus a fundamental quantity for future spin electronics, as it allows full electric manip- ulation of magnetic moments [26]. It has turned out that owing to spin-orbit interaction, one can generate pure spin current in otherwise nonmagnetic systems like graphene [27, 28], or nonmagnetic semiconductors, where the spin-orbit coupling is particularly strong [29]. This coupling leads to spin Hall effect, where perpendicular spin current is induced by an electric field. The spin Hall effect can be generated either by extrinsic (due to impurities) spin-orbit interaction or by intrinsic one. Spin currents can be also generated by other methods, like for instance spin thermoelectric effect [30].

\section{Spin torque and current-induced switching in metallic spin valves}

When orientation of local magnetic moments varies in space, a nonzero net spin (angular) momentum can be locally transferred from conduction electrons to local magnetic moments. This appears as a torque exerted locally on the magnetization. In spin valve structures the spin-transfer torque may lead to the phenomenon of current-induced magnetic switching (CIMS), predicted first theoretically $[7,8]$, and then confirmed experimentally $[9,10]$. The magnetic switching can be detected via resistance measurements owing to the GMR effect. The switching between low and high resistance states (parallel and antiparallel magnetic configurations) has been observed for currents exceeding certain critical values and for sufficiently low external fields. For larger fields, transition to steady precessional regime has been observed $[11,12]$.

A typical nanopillar spin valve consists of two magnetic films separated by a nonmagnetic one. One of the layers is thick and its magnetic moment is fixed (reference layer), while the second layer is thin and its magnetic moment is free to rotate under the influence of either magnetic field or current-induced spin torque. When magnetic moments of the two layers are noncollinear, spin 
orientation of an electron leaving a particular layer is not collinear with the spin quantization axis of the second layer. Consequently, the electron spin starts precessing due to exchange field, and becomes aligned along the quantization axis on the distance from the interface of the order of the corresponding Fermi wavelength [31]. Thus, the perpendicular component of the spin current is totally (or partly for ultrathin layers) absorbed by the layer. The absorbed spin momentum is equivalent to the torque $\tau$ exerted on the ferromagnetic film. This torque (per unit square) can be calculated as

$$
\tau=\frac{\hbar}{2}\left(j_{\perp \mathrm{L}}-\boldsymbol{j}_{\perp \mathrm{R}}\right)
$$

where $j_{\perp \mathrm{L}}$ and $\boldsymbol{j}_{\perp \mathrm{R}}$ are the normal to the magnetization components of the spin current density at the left and right interfaces of the magnetic film, taken on the normal metal side of these interfaces.

The torque is usually decomposed into two components: one in the plane formed by the two magnetic moments, and the other one normal to this plane,

$$
\boldsymbol{\tau}=a I \hat{\boldsymbol{s}} \times(\hat{\boldsymbol{s}} \times \hat{\boldsymbol{S}})+b I \hat{\boldsymbol{s}} \times \hat{\boldsymbol{S}},
$$

where $I$ is the charge current density, $\hat{\boldsymbol{s}}$ and $\hat{\boldsymbol{S}}$ are the unit vectors along the spin polarization of the sensing and reference magnetic layers, respectively, while $a$ and $b$ are some parameters. The first term (called sometimes Slonczewski's torque) is in the plane formed by magnetic moments of the two magnetic films, while the second component is perpendicular to this layer. In metallic spin valves, the parameter $b$ is usually one or two orders of magnitude smaller than $a$, so the normal component can be generally neglected, although it can play some role in spin dynamics. Let us note that spin torque changes sign when $I$ is reversed.

The spin torque due to spin transfer vanishes in collinear configurations, but is nonzero in noncollinear ones. Generally, behavior of the torque with the angle between magnetic moments depends on transport regime. One also should note that this angular dependence comes not only from the vector products in Eq. (2), but also from the angular dependence of the parameters $a$ and $b$. This angular dependence in the ballistic transport regime $[7,8]$ may be different from that in the diffusive transport [32].

Diffuse transport theory of electronic transport in spin valves is usually based on the macroscopic approach by Valet and Fert [33]. This approach was originally developed for GMR in collinear magnetic configurations, and later was extended to GMR in noncollinear configurations and also to spin torque $[32,34]$. An important feature of this approach is spin accumulation, which builds up at the interfaces due to the spin "bottle neck" effect. This theory is linear in the sense that spin accumulation and spin currents are linear in voltage (or in the charge current). Such linear model, however, does not describe some of recent experiments on nonlinear transport in double spin valves [35], so the Valet-Fert description was extended to include also some nonlinear effects following from the dependence of the key parameters of the model on charge current [36].

If positive current, $I>0$ (flowing from the reference towards the sensing layer), tends to destabilize the parallel configuration and switch to antiparallel one for current exceeding a certain threshold value, then a negative current usually tends to destabilize the antiparallel configuration and stabilize the parallel one $[9,10]$. Such a switching is called normal CIMS. In turn, when positive current induces transition to parallel configuration, it is called inverse CIMS. By reversing bias polarization one can also reverse the magnetic switching. It has been shown that the spin asymmetry factor of the reference layer determines whether the switching is normal or inverse; when this factor is positive, the switching is normal, and when it is negative, the switching is inverse [32].

In standard spin valves (those which show either normal or inverse current-induced switching in the absence of external magnetic field), transition to stationary precessional states is possible only in a sufficiently strong external magnetic field [12]. When current is above a certain critical value and tends to rotate magnetic moment of the sensing layer, while the applied magnetic field tends to restore its original orientation, competition of these two torques may lead to a stationary precessional state. Although the damping term (Gilbert damping) leads to energy dissipation, the energy is continuously pumped from the voltage source to the magnetic system and supports microwave oscillations. In some asymmetric structures (like $\mathrm{Co} / \mathrm{Cu} / \mathrm{Py}$ ), the angular dependence of spin torque in the diffusive transport regime exhibits some nonstandard behavior, i.e. the in-plane spin torque vanishes at a certain noncollinear configuration. Owing to this, transition to the precessional states can occur also in zero magnetic field [37, 38]. Such systems are of particular interest as they may be used as microwave nanogenerators entirely controlled by electric field.

To describe dynamical behavior of the sensing layer under the influence of external field and spin current, one can use the phenomenological Landau-Lifshitz-Gilbert equation, which additionally includes the torque due to spin transfer. Time evolution of a unit vector $\hat{\boldsymbol{s}}=$ $\left(s_{x}, s_{y}, s_{z}\right)$ along the spin moment of the sensing layer is then determined by

$$
\frac{\mathrm{d} \hat{\boldsymbol{s}}}{\mathrm{d} t}=-\left|\gamma_{\mathrm{g}}\right| \mu_{0} \hat{\boldsymbol{s}} \times \boldsymbol{H}_{\mathrm{eff}}-\alpha \hat{\boldsymbol{s}} \times \frac{\mathrm{d} \hat{\boldsymbol{s}}}{\mathrm{d} t}+\frac{\left|\gamma_{\mathrm{g}}\right|}{M_{\mathrm{s}} d} \boldsymbol{\tau},
$$

where $\gamma_{\mathrm{g}}$ is the gyromagnetic ratio, $\mu_{0}$ is the magnetic vacuum permeability, $M_{\mathrm{s}}$ is the saturation magnetization, $d$ is the sensing layer thickness, and $\alpha$ is the Gilbert damping parameter. The effective field $\boldsymbol{H}_{\text {eff }}$ includes contributions from external magnetic field $\left(\boldsymbol{H}_{\text {ext }}\right)$, uniaxial magnetic anisotropy $\left(\boldsymbol{H}_{\text {ani }}\right)$, and the demagnetization field $\left(\boldsymbol{H}_{\text {dem }}\right) ; \boldsymbol{H}_{\text {eff }}=\boldsymbol{H}_{\text {ext }}+\boldsymbol{H}_{\text {ani }}+\boldsymbol{H}_{\text {dem }}$. The damping parameter $\alpha$ is usually assumed to be constant, but this assumption is generally not justified as the damping may depend on the angle between magnetic moments. 


\section{Magnetic planar tunnel junctions}

Effect similar to the GMR also occurs when the nonmagnetic metallic spacer layer is replaced with a nonmagnetic insulating barrier. This effect was discovered long time ago in ferromagnetic planar junctions by Jullière [13], much earlier than the GMR effect in metallic multilayers was found. Currently, magnetic tunnel junctions are of great interest due to possible applications in magnetic storage technology (magnetic random access memories, MRAM). As in the case of GMR, the tunnel magnetoresistance (TMR) consists in a decrease (increase is also possible) in the junction resistance when magnetic configuration of the junction varies from antiparallel to parallel. One of the most promising junctions are those based on the $\mathrm{MgO}$ barrier, where the TMR of the order of several hundreds percent has been observed [39].

The TMR effect also exists in more complex tunnel junctions, like for instance double barrier planar ones with magnetic electrodes, or double barrier junctions with magnetic barriers and nonmagnetic metallic electrodes. In the latter case spin filtering by ferromagnetic barriers (eg. EuS) can lead to large values of TMR, as predicted theoretically [40] and then confirmed experimentally [41]. Physics of TMR effect is similar to that of GMR, although the role of various parameters and features of the electronic structures may be different.

Similarly to metallic spin valves, spin current accompanying charge current can lead to spin transfer torque and then to the CIMS phenomenon [14-16]. The normal component of the spin transfer torque, however, is now of the order of in-plane one [42, 43], which makes the current-induced magnetic dynamics more complex. What is important from the application point of view is the critical current that is now smaller by one or two orders of magnitude from the critical current in metallic spin valves.

\section{Molecular junctions}

The magnetoresistance phenomena and also current-induced magnetic switching are general phenomena. Spin current can be used to control and change magnetic state of any magnetic object, provided the torque is strong enough. In turn, to control magnetic state and switching process one can make use of the magnetoresistance phenomena. Now we discuss briefly the possibility of using spin current to reverse magnetic moment of a molecular magnet.

Single-molecule magnets (SMMs) attract much interest as they exhibit magnetic bistability required for memory elements [44-46]. However, magnetic state of the molecule is stable at very low temperatures, of the order of $1 \mathrm{~K}$. At higher temperatures SMMs behave like superparamagnetic particles. It has been shown that one can fully electronically control magnetic state of the molecule at low temperatures, when the molecule is attached to external ferromagnetic leads [25, 47, 48].
An SMM is a quantum object and any description of transport properties should take into account discrete energy levels and also Coulomb correlations of electrons in the molecule. In the sequential tunneling regime $[25,47,48]$, electronic transport occurs due to tunneling of electrons between the leads and the lowest unoccupied molecular orbital (LUMO) level of the SMM. The CIMS then appears due to exchange coupling of electrons in the LUMO level with the molecule's internal magnetic core. Hamiltonian describing the molecule can be presented in the form $[25,47]$

$$
\begin{aligned}
& \mathcal{H}_{\mathrm{SMM}}=-\left(D+\sum_{\sigma} D_{1} c_{\sigma}^{\dagger} c_{\sigma}+D_{2} c_{\uparrow}^{\dagger} c_{\uparrow} c_{\downarrow}^{\dagger} c_{\downarrow}\right) S_{z}^{2} \\
& +\sum_{\sigma} \varepsilon c_{\sigma}^{\dagger} c_{\sigma}+U c_{\uparrow}^{\dagger} c_{\uparrow} c_{\downarrow}^{\dagger} c_{\downarrow}-\frac{1}{2} J \sum_{\sigma \sigma^{\prime}} \sigma_{\sigma \sigma^{\prime}} \cdot \boldsymbol{S} c_{\sigma}^{\dagger} c_{\sigma^{\prime}} .
\end{aligned}
$$

The first term accounts for the uniaxial magnetic anisotropy of the molecule. Here $D$ is the anisotropy constant of an uncharged molecule, while $D_{1}$ and $D_{2}$ take into account the modification of the anisotropy constant when the LUMO level is occupied by one or two electrons, respectively. The operator $c_{\sigma}^{\dagger}\left(c_{\sigma}\right)$ creates (annihilates) an electron in the LUMO level of energy $\varepsilon$, whereas $\boldsymbol{S}$ is the molecule's core spin operator. The next two terms describe the LUMO level, where $U$ is the Coulomb energy of two electrons with opposite spins in the LUMO level. The last term represents the exchange interaction between the SMM core and electrons in the LUMO level, with $\sigma=\left(\sigma_{x}, \sigma_{y}, \sigma_{z}\right)$ denoting the Pauli matrices and $J$ being the relevant exchange parameter.

Mechanism of magnetic switching relies now on exchange interaction between electron flowing through the LUMO level and molecule's core. Owing to this interaction, spin of the electron becomes reversed, while magnetic state of the molecule's core becomes also changed. The total angular momentum, however, is conserved. Such processes randomize magnetic state in the case of nonmagnetic electrodes, while may lead to switching of the molecule's spin between two states of lowest energy when the electrodes are ferromagnetic [48].

\section{Spin currents}

From the above it is clear that spin current is the physical quantity which plays a crucial role in electric manipulation of magnetic moments. From the theoretical point of view, operator of spin current can be written in the form

$$
\hat{\jmath}_{i}^{\alpha}=\frac{1}{4 e}\left\{\hat{\jmath}_{i}, \sigma_{\alpha}\right\},
$$

where $\hat{\jmath}_{i}$ is the $i$-th component of the charge current operator $(i=x, y, z)$, and $\sigma_{\alpha}$ are the Pauli matrices $(\alpha=x, y, z)$. Therefore, it is important to have efficient methods of spin current generation. The easiest way is to make use of the fact that charge current in ferromagnetic metals/semiconductors is accompanied by a spin current due to asymmetry of the two spin channels for electronic transport. 
However, it is very important to heave methods of spin current generation in nonmagnetic metals/semiconductors. This can be achieved by several techniques, including injection from ferromagnetic metal/semiconductor to nonmagnetic systems, or using circularly polarized light to create conduction electrons of a particular spin polarization in semiconductors. In both these cases spin current is associated with a charge current. To get a pure spin current in nonmagnetic systems one may use also several techniques. One of them is based on a nonlocal nature of spin accumulation [26]. However, the most promising way to get pure spin currents is based on spin Hall effect, which exists in nonmagnetic systems exhibiting relatively strong spin-orbit interaction [27, 29]. Such spin-orbit interaction can be either due to impurities or it can be of intrinsic origin.

The spin Hall effect has been proven to exist in many systems. Of particular interest is spin Hall effect in two-dimensional electron gas with Rashba spin-orbit interaction [29], where the spin Hall conductivity has been shown to be universal. Unfortunately, the presence of impurities and potential (independent of electron spin) scattering of electrons turned out to suppress the spin Hall effect. The spin Hall effect has been confirmed in a number of experiments [49-51], and is currently a subject of extensive theoretical and experimental investigations.

\section{Acknowledgments}

This work was supported by funds from the Ministry of Science and Higher Education as research project in years 2007-2010.

\section{References}

[1] P. Grünberg, R. Schreiber, Y. Pang, M.B. Brodsky, H. Sowers, Phys. Rev. Lett. 57, 2442 (1986).

[2] P. Bruno, C. Chappert, Phys. Rev. Lett. 67, 1602 (1991).

[3] J. Barnaś, J. Magn. Magn. Mater. 111, L215 (1992).

[4] M.N. Baibich, J.M. Broto, A. Fert, F.N. Van Dau, F. Petroff, P.Etienne, , G. Creuzet, A. Friederich, J. Chazelas, Phys. Rev. Lett. 61, 2472 (1988).

[5] G. Binasch, P. Grünberg, F. Saurenbach, W. Zinn, Phys. Rev. B 39, 4828 (1989).

[6] J. Barnaś, A. Fuss, R.E. Camley, P. Grünberg, W. Zinn, Phys. Rev. B 42, 8110 (1990); R.E. Camley, J. Barnaś, Phys. Rev. Lett. 63, 664 (1989).

[7] J.C. Slonczewski, J. Magn. Magn. Mater. 159, L1 (1996); 195, L261 (1999).

[8] L. Berger, Phys. Rev. B 54, 9353 (1996).

[9] J.A. Katine, F.J. Albert, R.A. Buhrman, E.B. Myers, D.C. Ralph, Phys. Rev. Lett. 84, 3149 (2000).

[10] M. AlHajDarwish, H. Kurt, S. Urazhdin, A. Fert, R. Loloee, W.P. Pratt, Jr., J. Bass, Phys. Rev. Lett. 93, 157203 (2004).

[11] S.I. Kiselev, J.C. Sankey, I.N. Krivorotov, N.C. Emley, R.J. Schoelkopf, R.A. Buhrman, D.C. Ralph, $\mathrm{Na}$ ture 425, 380 (2003).
[12] I.N. Krivorotov, N.C. Emley, J.C. Sankey, S.I. Kiselev, D.C. Ralph, R.A. Buhrman, Science 307, 228 (2005).

[13] M. Jullière, Phys. Lett. A 54, 225 (1975).

[14] Y. Huai, F. Albert, P. Nguyen, M. Pekala, T. Valet, Appl. Phys. Lett. 84, 3118 (2004).

[15] J. Haykawa, S. Ikeda, Y.M. Lee, R. Sasaki, T. Meguro, F. Matsukura, H. Takahashi, H. Ohno, Jpn. J. Appl. Phys. 45, L1057 (2006).

[16] J.C. Sankey, Y.-T. Cui, J.Z. Sun, J.C. Slonczewski, R.A. Buhrman, D.C. Ralph, Nature Phys. 4, 67 (2008).

[17] H. Kubota, A. Fukushima, K. Yakushiji, T. Nagahama, S. Yuasa, K. Ando, H. Maehara, Y. Nagamine, K. Tsunekawa, D.D. Djayaprawira, N. Watanabe, Y. Suzuki, Nature Phys. 4, 37 (2008).

[18] M. Wilczyński, R. Świrkowicz, J. Barnaś, Acta Phys. Pol. A 115, 269 (2009).

[19] K. Ono, H. Shimada, S. Kobayashi, Y. Outuka, J. Phys. Soc. Japan 65, 3449 (1996).

[20] J. Barnaś, A. Fert, Phys. Rev. Lett. 80, 1058 (1998).

[21] M.M. Deshmukh, D.C. Ralph, Phys. Rev. Lett. 89, 266803 (2002).

[22] K. Hamaya, S. Masubuchi, M. Kawamura, T. Machida, M. Jung, K. Shibata, K. Hirakawa, T. Taniyama, S. Ishida, Y. Arakawa, Appl. Phys. Lett. 90, 053108 (2007).

[23] J. Barnaś, I. Weymann, J. Phys., Condens. Matter 20, 423202 (2008) (Topical Review).

[24] K. Tsukagoshi, B.W. Alphenaar, H. Ago, Nature 401, 572 (1999).

[25] M. Misiorny, J. Barnaś, Phys. Rev. B 75, 134425 (2007); ibid 76, 54448 (2007).

[26] T. Kimura, Y. Otani, J. Hamrle, Phys. Rev. Lett. 96, 037201 (2006).

[27] C.L. Kane, E.J. Mele, Phys. Rev. Lett. 95, 226801 (2005).

[28] A. Dyrdał, V.K. Dugaev, J. Barnaś, Phys. Rev. B 80, 155444 (2009).

[29] J. Sinova, D. Culcer, Q. Niu, N.A. Sinitsyn, T. Jungwirth, A.H. MacDonald, Phys. Rev. Lett. 92, 126603 (2004).

[30] K. Uchida, S. Takahashi, K. Harii, J. Ieda, W. Koshibae, K. Ando, S. Maekawa, E. Saitoh, $\mathrm{Na}$ ture 455, 778 (2008).

[31] M.D. Stiles, A. Zangwill, Phys. Rev. B 66, 014407 (2002); J. Appl. Phys. 91, 6812 (2002).

[32] J. Barnaś, A. Fert, M. Gmitra, I. Weymann, V.K. Dugaev, Phys. Rev. B 72, 024426 (2005).

[33] T. Valet, A. Fert, Phys. Rev. B 48, 7099 (1993).

[34] M. Gmitra, J. Barnaś, Phys. Rev. B 79, 12403 (2009).

[35] A. Aziz, O.P. Wessely, M. Ali, D.M. Edwards, C.H. Marrows, B.J. Hickey, M.G. Blamire, Phys. Rev. Lett. 103, 237203 (2009).

[36] P. Balaz, J. Barnaś, to be published.

[37] M. Gmitra, J. Barnaś, Phys. Rev. Lett. 96, 207205 (2006). 
[38] O. Boulle, V. Cros, J. Grollier, L.G. Pereira, C. Deranlot, F. Petro, G. Faini, J. Barnaś, A. Fert, Nature Phys. 3, 492 (2007).

[39] S. Yuasa, T. Nagahama, A. Fukushima, Y. Suzuki, K. Ando, Nature Mater. 3, 868 (2004).

[40] M. Wilczyński, J. Barnaś, Sensors Actuators A 91, 188 (2001).

[41] G.-X. Miao, M. Müller, J.S. Moodera, Phys. Rev. Lett. 102, 076601 (2009).

[42] I. Theodonis, N. Kioussis, A. Kalitsov, M. Chshiev, W.H. Butler, Phys. Rev. Lett. 97, 237205 (2006); A. Kalitsov, I. Theodonis, N. Kioussis, M. Chshiev, W.H. Butler, A. Vedyayev, J. Appl. Phys. 99, 086501 (2006); I. Theodonis, A. Kalitsov, N. Kioussis, Phys. Rev. B 76, 224406 (2007).

[43] M. Wilczyński, J. Barnaś, R. Świrkowicz, Phys. Rev. B 77, 1 (2008).
[44] R. Sessoli, D. Gatteschi, A. Caneschi, M.A. Novak, Nature 365, 141 (1993).

[45] C. Joachim, J.K. Gimzewski, A. Aviram, Nature 408, $541(2000)$.

[46] L. Bogani, W. Wernsdorfer, Nature Mater. 7, 179 (2008).

[47] C. Timm, F. Elste, Phys. Rev. B 73, 235304 (2006).

[48] M. Misiorny, J. Barnaś, Phys. Status Solidi B 246, 695 (2009).

[49] S. Murakami, N. Nagaosa, S.C. Zhang, Science 301, 1348 (2003).

[50] Y.K. Kato, R.C. Myers, A.C. Gossard, D.D. Awschalom, Science 306, 1910 (2004).

[51] J. Wunderlich, B. Kaestner, J. Sinova, T. Jungwirth, Phys. Rev. Lett. 94, 047204 (2005). 